SVN - 1 Neurology

To cite: Yang G-Y. Advancement in stroke research. Stroke and Vascular Neurology 2019;4: e000253. doi:10.1136/svn2019-000253

Received 30 May 2019 Accepted 30 May 2019 Published Online First 23 June 2019
Check for updates

(c) Author(s) (or their employer(s)) 2019. Re-use permitted under CC BY-NC. No commercial re-use. See rights and permissions. Published by BMJ.

Neuroscience and Neuroengineering Center, Med-X Research Institute and School of Biomedical Engineering, Shanghai Jiao Tong University, Shanghai, China

Correspondence to Dr Guo-Yuan Yang; gyyang0626@163.com

\title{
Advancement in stroke research
}

\author{
Guo-Yuan Yang
}

Stroke is the second most common cause of death in the world especially in the ageing population. ${ }^{1}$ Thrombolysis and intravascular embolectomy are the main treatment and populated rapidly. ${ }^{2}{ }^{3}$ However, the beneficiary is still limited because the mechanistic studies are behind. The basic research on stroke is improved because of new devices, novel techniques and the development of other disciplines. ${ }^{4}$ The update of a broad range of topics related to stroke is a widely appreciated feature. In order to improve the understanding of cellular and molecular mechanisms of stroke, the 2nd Summit of Chinese Basic Science Research on Stroke 2018 has been held in Beijing, China. Total of 60 basic stroke researchers from China and abroad attended this meeting. The meeting focused on six fields: (1) neuroprotection in acute ischaemic stroke; (2) neurorepair and regeneration; (3) neuroinflammation and neuroimmunology; (4) cognitive disorder in stroke; (5) drug/small molecule therapy for stroke; and (6) advances in optical imaging.

Although $95 \%$ of published preclinical studies on 'neuroprotective agents' (19902018) reported positive outcomes in animal models of ischaemic stroke, none translated to successful phase III trials. ${ }^{5}$ Studies are therefore focusing other approaches. For example, preconditioning and postconditioning induced neuroprotection are advancements in our understanding of the neurochemical basis for their neuroprotective role. ${ }^{6}$ Some of these studies are trying to transfer these techniques to the clinical practice. In the current issue, antioxidant agents such as hydrogen sulfide is another hot topic of neuroprotection. Numerous studies show that hydrogen sulfide impacts stroke outcomes in animal and cellular models. This action is via upregulation of the endothelium-dependent contraction and dilation function of cerebral vessels although having controversial results. ${ }^{7}$ In addition, Wang et al provided the direct evidence that nicotinamide phosphoribosyltransferase could be a promising therapeutic target for ischaemic stroke, which has been published in this issue. It is interesting that the effect and mechanism of dl-3-N-butylphthalide (NBP), a new anti-ischaemic stroke drug developed by Chinese researchers, has been discussed by Wang et al. ${ }^{8}$ NBP could affect local blood flow and improve microcirculation during ischaemia. ${ }^{9}{ }^{10}$ This discussion will promote and extend the use of NBP from the acute ischaemic stroke to the chronic ischaemic brain injury.

Neuroinflammation is one of the hot topics in the study of mechanism of ischaemic stroke. Microglia activation, polarisation and specific functions during the different periods of stroke have been extensively studied. ${ }^{11}$ Confocal microscope or two-photon imaging techniques can now demonstrate the factors between microglial activation and ischaemia severity. In current issue, activated microglia displayed diverse polarisation phenotypes and played different functions during postischaemia repair and remodelling. Studies have found that microRNA playedan important role in inflammatory response during ischaemic brain injury. Furthermore, Shen et al reported the relationship between non-coding RNA and neuroinflammatory response. A potential role of circRNAs in neuroinflammation and neuron functional recovery during ischaemic stroke remains unknown. Shen et al discussed the function of circRNAs, including circHECTD1 and circDLGAP4, in the experimental ischaemic research, which may become biomarkers and therapeutic targets for the stroke therapy. ${ }^{12} 13$

In this issue, regarding the research of blood-brain barrier, Chopp's group studied the glymphatic system using neuroimaging and immunohistochemistry techniques. Glymphatic system is a newly discovered pseudolymphatic system in the brain, which plays an important role in the removal of interstitial metabolic waste products. MRI could dynamically detect the role of glymphatic system in neurological diseases, its potential of pathways and function of modelling and so on. Further study found that diabetes mellitus and ageing could impair glymphatic system, leading to metabolic waste accumulation in the injured brain and consequently inducing cognitive dysfunction. The majority of blood-brain barrier research focuses on 
its cellular constituents while the basement membrane is less studied. In this issue, Xu et al focused on the role of the basement membrane in blood-brain barrier integrity, especially the major components of basement membrane including collagen IV, laminin, nidogen and perlecan, which was synthesised predominantly by brain microvascular endothelial cells, pericytes and astrocytes.

The occurrence of haemorrhagic stroke was relatively less compared with that of the ischaemic stroke. However, the mortality and morbidity of haemorrhagic stroke are higher. Mass factor, brain oedema and blood-brain barrier disruption are often studied in the haemorrhagic stroke both in experimental and clinical field; however, the results are not optimistic. ${ }^{14}$ The knowledge of postintracerebral haemorrhage neuronal death and the molecular mechanism is largely unknown. Recently, an iron-dependent form of non-apoptotic cell death known as ferroptosis has been identified. In this issue, Wan et al demonstrated that ferroptosis occurred after experimental haemorrhagic stroke model both in vivo and in vitro conditions and contributed to the neuronal death. They summarised that current research on iron toxicity, lipid peroxidation and ferroptosis in intracerebral haemorrhage and the underlying molecular mechanisms of ferroptosis.

Brain arteriovenous malformation (BAVM) and cerebral cavernous malformations (CCMs) often cause intracranial haemorrhage. ${ }^{15}$ Understanding the genetic abnormality and molecular mechanism of cerebral vascular diseases are extremely important. BAVM and CCMs could increase risk of haemorrhage, focal neurological defects and seizures. Studies have demonstrated that three different genes are associated with CCMs' progression, and CCM3 mutation results in a more severe form of the disease. In current issue, Wang et al focused on the signalling pathways mediated by CCM3 in regulating endothelial cell junction, proliferation, migration and permeability.

Cerebral small vessel disease (CSVD) was the main cause of vascular dementia. The diagnosis of CSVD is generally based on clinical symptoms or imaging features, which is controversial. In this issue, Han et $a l^{33}$ reviewed the current knowledge of pathological mechanisms of microvascular dysfunction and neurodegeneration in dementia and particularly focused on oxidative/nitrosative stress, cellular metabolic dysfunction, inflammatory signalling, abnormal synaptic plasticity and its implications in dementia. Advanced multimodal neuroimaging techniques such as brain network connectome analyses are currently applied to explore the underlying mechanism of vascular dementia. The knowledge of structural and functional brain network in a poststroke longitudinal
CSVD cohort was progressive, which has been published in this issue. The result showed that brain structural network disruption may play a pivot role in the cognitive decline.

\section{Contributors solo author.}

Funding The authors have not declared a specific grant for this research from any funding agency in the public, commercial or not-for-profit sectors.

Competing interests None declared.

Patient consent for publication Not required.

Provenance and peer review Commissioned; internally peer reviewed.

Open access This is an open access article distributed in accordance with the Creative Commons Attribution Non Commercial (CC BY-NC 4.0) license, which permits others to distribute, remix, adapt, build upon this work non-commercially, and license their derivative works on different terms, provided the original work is properly cited, appropriate credit is given, any changes made indicated, and the use is non-commercial. See: http://creativecommons.org/licenses/by-nc/4.0/.

\section{REFERENCES}

1. Wang W, Jiang B, Sun $\mathrm{H}$, et al. Prevalence, Incidence, and Mortality of Stroke in China: Results from a Nationwide Population-Based Survey of 480687 Adults. Circulation 2017:135:759-71.

2. Cheng NT, Kim AS. Intravenous Thrombolysis for Acute Ischemic Stroke Within 3 Hours Versus Between 3 and 4.5 Hours of Symptom Onset. Neurohospitalist 2015;5:101-9.

3. Goyal M, Demchuk AM, Menon BK, et al. Randomized assessment of rapid endovascular treatment of ischemic stroke. N Engl J Med 2015;372:1019-30

4. Bosetti F, Koenig Jl, Ayata C, et al. Translational Stroke Research: Vision and Opportunities. Stroke 2017;48:2632-7.

5. Philip M, Benatar M, Fisher M, et al. Methodological quality of animal studies of neuroprotective agents currently in phase II/III acute ischemic stroke trials. Stroke 2009;40:577-81.

6. Li S, Hafeez A, Noorulla F, et al. Preconditioning in neuroprotection: From hypoxia to ischemia. Prog Neurobiol 2017;157:79-91.

7. Bredthauer A, Lehle K, Scheuerle A, et al. Intravenous hydrogen sulfide does not induce neuroprotection after aortic balloon occlusion-induced spinal cord ischemia/reperfusion injury in a human-like porcine model of ubiquitous arteriosclerosis. Intensive Care Med Exp 2018;6:44.

8. Wang S, Ma F, Huang L, et al. DI-3-n-Butylphthalide (NBP): A Promising Therapeutic Agent for Ischemic Stroke. CNS Neurol Disord Drug Targets 2018;17:338-47.

9. Qin C, Zhou P, Wang L, et al. DI-3-N-butylphthalide attenuates ischemic reperfusion injury by improving the function of cerebral artery and circulation. J Cereb Blood Flow Metab 2018;271678X:027 1678X1877683.

10. Zhou PT, Wang LP, Qu MJ, et al. DI-3-N-butylphthalide promotes angiogenesis and upregulates sonic hedgehog expression after cerebral ischemia in rats. CNS Neurosci Ther 2019;25:748-58.

11. Ma $Y$, Wang J, Wang $Y$, et al. The biphasic function of microglia in ischemic stroke. Prog Neurobiol 2017;157:247-72.

12. Bai $Y$, Zhang $Y$, Han B, et al. Circular RNA DLGAP4 Ameliorates Ischemic Stroke Outcomes by Targeting miR-143 to Regulate Endothelial-Mesenchymal Transition Associated with Blood-Brain Barrier Integrity. J Neurosci 2018;38:32-50.

13. Han $B$, Zhang $Y$, Zhang $Y$, et al. Novel insight into circular RNA HECTD1 in astrocyte activation via autophagy by targeting MIR142TIPARP: implications for cerebral ischemic stroke. Autophagy 2018;14:1164-84.

14. Xi G, Keep RF, Hoff JT. Mechanisms of brain injury after intracerebral haemorrhage. Lancet Neurol 2006;5:53-63.

15. Cavalcanti DD, Kalani MY, Martirosyan NL, et al. Cerebral cavernous malformations: from genes to proteins to disease. J Neurosurg 2012;116:122-32. 